\title{
Wavelet-Based Methodology for Evolutionary Spectra Estimation of Nonstationary Typhoon Processes
}

\author{
Guang-Dong Zhou, ${ }^{1}$ You-Liang Ding, ${ }^{2}$ and $\mathrm{Ai}-\mathrm{Qun} \mathrm{Li}^{2}$ \\ ${ }^{1}$ College of Civil and Transportation Engineering, Hohai University, Nanjing 210098, China \\ ${ }^{2}$ China Key Laboratory of Concrete and Prestressed Concrete Structures of Ministry of Education, Southeast University, \\ Nanjing 210096, China \\ Correspondence should be addressed to Guang-Dong Zhou; zhougd@hhu.edu.cn
}

Received 14 August 2014; Accepted 9 October 2014

Academic Editor: Ting-Hua Yi

Copyright ( 92015 Guang-Dong Zhou et al. This is an open access article distributed under the Creative Commons Attribution License, which permits unrestricted use, distribution, and reproduction in any medium, provided the original work is properly cited.

Closed-form expressions are proposed to estimate the evolutionary power spectral density (EPSD) of nonstationary typhoon processes by employing the wavelet transform. Relying on the definition of the EPSD and the concept of the wavelet transform, wavelet coefficients of a nonstationary typhoon process at a certain time instant are interpreted as the Fourier transform of a new nonstationary oscillatory process, whose modulating function is equal to the modulating function of the nonstationary typhoon process multiplied by the wavelet function in time domain. Then, the EPSD of nonstationary typhoon processes is deduced in a closed form and is formulated as a weighted sum of the squared moduli of time-dependent wavelet functions. The weighted coefficients are frequency-dependent functions defined by the wavelet coefficients of the nonstationary typhoon process and the overlapping area of two shifted wavelets. Compared with the EPSD, defined by a sum of the squared moduli of the wavelets in frequency domain in literature, this paper provides an EPSD estimation method in time domain. The theoretical results are verified by uniformly modulated nonstationary typhoon processes and non-uniformly modulated nonstationary typhoon processes.

\section{Introduction}

The wind-induced dynamic responses are important factors that must be taken into account during the design and assessment of long-span bridges, tall buildings, and other large-scale structures above ground or sea level with low stiffness and damping. In order to exactly predict structural wind-induced vibration, comprehensively understanding wind properties is the prior work. Traditional concepts treat the wind as a realization of a stationary stochastic process, whose statistical moments and frequency content keep constants during the entire duration. However, due to the energy dissipation when it attacks a structure, sudden and sporadic behaviors exhibit in many field monitoring typhoon processes. Therefore, it is more realistic to model typhoon winds as nonstationary processes, whose amplitudes, mean values, and frequency content change in time $[1,2]$. Clearly, nonstationary features cannot be captured by conventional spectra estimation methods such as the Fourier transform, the Welch method, and the modern spectra estimation technology since these methods only calculate the mean composition of the power spectral density during the whole process.

In the past decades, intensive research efforts have been made to describe the time-dependent spectral characteristics of different nonstationary processes and many methods have been developed, such as the Wigner-Ville method (WVM), the short-time Fourier transform (STFT), and the S-transform (ST). The WVM defines a time-dependent spectrum at time $t$ as the Fourier transform of an instantaneous correlation function $R(\tau, t)$, which corresponds to the standard correlation function $R(\tau)$ with time lag $\tau$ centered at time $t$ [3-5]. The STFT applies a moveable narrow window to establish a local spectrum for any time instant 
based on the fast Fourier transform [2]. The ST, which is a generalization of the STFT, employs the scalable Gaussian window to view the local frequency content of nonstationary signals [6,7]. Unfortunately, these methods enclose some intrinsic drawbacks. The WVM cannot reflect the actual local behavior of the process at time $t$ since the variable $\tau$ must be integrated over an infinite range to compute the Fourier transform of the instantaneous correlation function $R(\tau, t)$. The STFT cannot achieve high resolutions in both time and frequency domains simultaneously due to the inherent limitation of the Fourier transform. And the ST may miss high frequency energy and it requires highly complex computation.

In practical application, such as investigating nonstationary features of the typhoon process, high resolution in both time and frequency domains is desired. To overcome the intrinsic shortcomings of existing nonstationary signal processing technologies, the wavelet transform has been developed. The wavelet transform has the capability of focusing on multiscale details of a signal by a series of moveable and scalable time-frequency windows, which are named as wavelets, and provides a promising tool to explore signal features in both time and frequency domains $[8,9]$. Applications of the wavelet transform to engineering problems can be found in a large number of studies that relate to signal processing [10-12], dynamic analysis of structures [13, 14], system identification [15-17], and damage detection [18-20]. Recently, wavelet analysis is getting utilized as a method of transforming time-sequential data to data on a time-frequency plane in civil engineering. Basu and Gupta have introduced closed-form expressions of waveletbased spectra by modified Littlewood-Paley wavelets for pursuing accurate time-dependent spectra of seismic records $[21,22]$. Iyama and Kuwamura have also proposed a timedependent spectrum based on nonorthogonal wavelets under restrictive assumptions [23]. However, in these studies, the computed time-dependent spectrum has been introduced in a somewhat heuristic manner as the local contribution to the mean-square value of the Fourier transform of the process and no rigorous treatment has been presented. In 2004, an innovated wavelet-based method was developed by Spanos and Failla to estimate the evolutionary power spectral density (EPSD) of a specific class of Priestley's oscillatory processes, such as seismic motions and fluctuating winds [24]. In this work, the rigorous derivation has been conducted and the EPSD is represented as a sum of time-independent shape functions, which is equal to the squared moduli of the Fourier transforms of the wavelets associated with the selected scales, modulated by timedependent coefficients. Subsequently, the method has been extended to arbitrary nonstationary processes by Failla et al. [5]

The method developed by Spanos and Failla provides a desirable accuracy to estimate the EPSD of Priestley's oscillatory processes and is effective in a large number of wavelet functions, including those which are not orthogonal in frequency domain. However, this method has an intrinsic drawback that it is inapplicable to those wavelets whose analytical expressions in frequency domain are not existent since the EPSD is formulated by the closed-form expressions of wavelet in frequency domain. In this paper, the EPSD of nonstationary typhoon processes is deduced and constructed by closed-form representations of wavelets in time domain, which can break the restriction in the method proposed by Spanos and Failla that the analytical frequency-dependent wavelet functions are required. And then, the reliability of the proposed method is demonstrated by uniformly modulated nonstationary typhoon processes and non-uniformly modulated nonstationary typhoon processes.

\section{Wavelet-Based Evolutionary Spectra Estimation}

2.1. Brief Description of the Wavelet Transform. The wavelet transform is a linear transform, which decomposes a signal via basis functions that are simply generated by scaling and shifting the "mother" wavelet through the convolution of the signal [25]. Scaling allows the time duration of the nonzero portion of the wavelet to be adjusted according to the local frequency content of the signal. And shifting enables the wavelet to observe the local frequency content of the signal at different time instant. The basis functions $\psi_{a, b}(t)$ originate from the "mother" wavelet $\psi(t)$ by

$$
\psi_{a, b}(t)=\frac{1}{\sqrt{a}} \psi\left(\frac{t-b}{a}\right) \quad(a, b \in R, a>0),
$$

where $a$ and $b$ represent the scale parameter and shift parameter, respectively.

The continuous wavelet transform (CWT) of a finite energy stochastic process $f(t)$ is defined as

$$
\begin{aligned}
\mathrm{WT}_{f}(a, b) & =\left\langle f(t), \psi_{a, b}(t)\right\rangle \\
& =\frac{1}{\sqrt{a}} \int_{-\infty}^{+\infty} f(t) \psi^{*}\left(\frac{t-b}{a}\right) d t,
\end{aligned}
$$

where $\mathrm{WT}_{f}(a, b)$ is the wavelet coefficient, $\langle\cdot\rangle$ means the convolution, and the symbol $(*)$ represents the complex conjugate.

In numerical analysis, the discrete version of wavelet transform is generally employed. The scale parameter $a$ and the shift parameter $b$ are discretized as

$$
\begin{aligned}
& a_{r}=a_{0}^{r} \quad r=1,2, \ldots m \\
& b_{k}=(k-1) b_{0} \quad k=1,2, \ldots n,
\end{aligned}
$$

where $a_{0}$ and $b_{0}$ are constants, $m$ represents the total number of discrete scales, and $n$ denotes the total number of time intervals retained in the discrete process $f(t)$.

2.2. Brief Description of the Evolutionary Power Spectral Density. Because of the complexity of nonstationary stochastic processes, defining a universal time-dependent spectrum for arbitrary nonstationary processes is impracticable. The EPSD, which is introduced by Priestley [26], is a time-dependent analogue of the spectrum of a stationary stochastic process 
and valid for a class of nonstationary oscillatory processes. The EPSD describes the local power-frequency distribution at each time instant, so it preserves the physical interpretation of the spectrum as a power-frequency distribution of a stationary stochastic process and is applied in many fields [27]. After extracting the time-varying mean wind speed, the remaining fluctuating wind speed of the typhoon process can be represented by a zero-mean nonstationary oscillatory process [1]. Therefore, the EPSD is introduced to describe the time-frequency features of the fluctuating component of the typhoon process.

Regarding an arbitrary stationary stochastic process $g(t)$, its spectral decomposition equation can be expressed as

$$
g(t)=\int_{-\infty}^{\infty} e^{i \omega t} d Z(\omega),
$$

where $i$ represents the imaginary unit and $Z(\omega)$ denotes a complex random process with orthogonal increments.

Since the spectral decomposition equation as (4) of nonstationary processes is not existent, the nonstationary stochastic process $f(t)$ can be represented by the following Riemann-Stieltjes (R-S) integration:

$$
f(t)=\int_{-\infty}^{+\infty} H(\omega, t) e^{i \omega t} d Z(\omega)
$$

where $H(\omega, t)$ denotes the slowly varying time- and frequency-dependent modulating function, namely, non-uniformly modulating function.

Then, the EPSD of the nonstationary stochastic process $f(t)$ is defined as

$$
S_{f f}(\omega, t)=|H(\omega, t)|^{2} S_{g g}(\omega),
$$

where $S_{f f}(\omega, t)$ is the EPSD of the process $f(t)$ and $S_{g g}(\omega)$ is the time-independent power spectral density of $g(t)$. It can be seen from (6) that, by using the modulating function, the time-independent power spectral density $S_{g g}(\omega)$ defined only in frequency domain is extended to three-dimensional space with time, frequency, and amplitude.

In engineering application, the non-uniformly modulating function is often replaced by a slowly varying uniformly modulating function $h(t)$, and (5) and (6) are simplified to

$$
\begin{gathered}
f(t)=\int_{-\infty}^{+\infty} H(\omega, t) e^{i \omega t} d Z(\omega)=h(t) g(t), \\
S_{f f}(\omega, t)=|H(\omega, t)|^{2} S_{g g}(\omega)=|h(t)|^{2} S_{g g}(\omega) .
\end{gathered}
$$

2.3. Wavelet-Based EPSD Estimation. According to the definition of the Fourier transform, the Fourier transform of $f(t)$ is

$$
F(\omega)=\int_{-\infty}^{\infty} f(t) e^{-i \omega t} d t
$$

where $F(\omega)$ represents the Fourier transform of $f(t)$. Substituting (5) into (8) results in

$$
F(\omega)=\int_{-\infty}^{\infty}\left[\int_{-\infty}^{\infty} H(\omega, t) e^{i \omega t} d Z(\omega)\right] e^{-i \omega t} d t
$$

Employing the definition of CWT in frequency domain, the CWT of $f(t)$ is

$$
\begin{aligned}
& \mathrm{WT}_{f}(a, b) \\
&=\frac{\sqrt{a}}{2 \pi} \int_{-\infty}^{\infty}\left\{\int_{-\infty}^{\infty}\left[\int_{-\infty}^{\infty} H(\omega, t) e^{i \omega t} d Z(\omega)\right] e^{-i \omega t} d t\right\} \\
& \times \Psi^{*}(a \omega) e^{i \omega b} d \omega .
\end{aligned}
$$

Since the uniformly modulating functions of practical signals in civil engineering vary slowly and the wavelets in frequency domain decay quickly at the vicinity of the central frequency, it can be assumed that the scales $a$ have one-to-one corresponding relation with the frequency $\omega$ in the wavelet transform. Thus, (5) and (6) can be approximately rewritten in the form

$$
\begin{gathered}
f(t) \approx \int_{-\infty}^{\infty} H(a, t) e^{i a t} d Z(a), \\
S_{f f}(a, t) \approx|H(a, t)|^{2} S_{g g}(a) .
\end{gathered}
$$

Then, the following equation can be obtained as follows:

$$
\begin{aligned}
& \mathrm{WT}_{f}(a, b) \\
& \approx \frac{\sqrt{a}}{2 \pi} \int_{-\infty}^{+\infty}\left\{\int_{-\infty}^{+\infty}\left[\int_{-\infty}^{+\infty} H(a, t) e^{i a t} d Z(a)\right] e^{-i \omega t} d t\right\} \\
& \times \Psi^{*}(a \omega) e^{i \omega b} d \omega .
\end{aligned}
$$

It is worth noting that (12) becomes more accurate if the change rate of the modulation function $H(a, t)$ is smaller than that of the wavelet functions in frequency domain. At a given time instant $b=b_{k}$, the wavelet transform of the process $f(t)$ is

$$
\begin{aligned}
& \mathrm{WT}_{f}\left(a, b_{k}\right) \\
& \begin{aligned}
& \approx \frac{\sqrt{a}}{2 \pi} \int_{-\infty}^{+\infty}\left\{\int_{-\infty}^{+\infty}\left[\int_{-\infty}^{+\infty} H(a, t) e^{i a t} d Z(a)\right] e^{-i \omega t} d t\right\} \\
& \times \Psi^{*}(a \omega) e^{i \omega b_{k}} d \omega \\
&=\int_{-\infty}^{\infty}\left\{\int_{-\infty}^{\infty}\left[H(a, t) \frac{\sqrt{a}}{2 \pi} \int_{-\infty}^{\infty} \Psi^{*}(a \omega) e^{-i \omega\left(t-b_{k}\right)} d \omega\right]\right. \\
&\left.\times e^{i a t} e^{i a t} d Z(a)\right\} e^{-i a t} d t .
\end{aligned}
\end{aligned}
$$

It can be found that the item $(\sqrt{a} / 2 \pi) \int_{-\infty}^{\infty} \Psi^{*}(a \omega) e^{-i \omega\left(t-b_{k}\right)} d \omega$ in (13) is the inverse Fourier transform of the wavelet function at time instant $b_{k}$. That is,

$$
\frac{\sqrt{a}}{2 \pi} \int_{-\infty}^{+\infty} \Psi^{*}(a \omega) e^{-i \omega\left(t-b_{k}\right)} d \omega=\frac{1}{\sqrt{a}} \psi\left(-\frac{t-b_{k}}{a}\right) .
$$


Substituting (14) into (13), the following equation can be derived:

$$
\begin{aligned}
\mathrm{WT}_{f}\left(a, b_{k}\right) & \\
=\int_{-\infty}^{\infty} & \left\{\int_{-\infty}^{\infty}\left[H(a, t) \frac{1}{\sqrt{a}} \psi\left(-\frac{t-b_{k}}{a}\right)\right] e^{i a t} e^{i a t} d Z(a)\right\} \\
\times & e^{-i a t} d t .
\end{aligned}
$$

Define a new nonstationary stochastic process $p(t)$, whose R$S$ integration is

$$
p(t)=\int_{-\infty}^{+\infty} D(a, t) e^{i a t} d Z(a),
$$

where $D(a, t)$ represents the non-uniformly modulating function of $p(t)$, which is

$$
D(a, t)=H(a, t) \frac{1}{\sqrt{a}} \psi\left(-\frac{t-b_{k}}{a}\right) e^{i a t} .
$$

Then, (15) can be simplified as

$$
\mathrm{WT}_{f}\left(a, b_{k}\right)=\int_{-\infty}^{+\infty} p(t) e^{-i a t} d t .
$$

Therefore, the wavelet coefficients, at a given instant $b_{k}$, $\mathrm{WT}_{f}\left(a, b_{k}\right)$, can be interpreted as the Fourier transform of $p(t)$, which is a new nonstationary stochastic process defined by the non-uniformly modulating function $D(a, t)$. The $D(a, t)$ is determined by (17), which indicates that $D(a, t)$ is equal to the wavelet function in time domain multiplied by the non-uniformly modulating function $H(a, t)$ of the original nonstationary stochastic process $f(t)$. According to (6), the EPSD of the nonstationary stochastic process $p(t)$ is

$$
\begin{aligned}
S_{p p}(a, t) & =|D(a, t)|^{2} S_{g g}(a) \\
& =|H(a, t)|^{2}\left|\frac{1}{\sqrt{a}} \psi\left(-\frac{t-b_{k}}{a}\right)\right|^{2} S_{g g}(a) \\
& =\left|\frac{1}{\sqrt{a}} \psi\left(-\frac{t-b_{k}}{a}\right)\right|^{2} S_{f f}(a) .
\end{aligned}
$$

Using the marginal property of the EPSD, that the integral of EPSD with time is equal to the square of the timeindependent power spectral density, the following equation can be obtained:

$$
\begin{aligned}
\left|\mathrm{WT}_{f}\left(a, b_{k}\right)\right|^{2} & =\int_{-\infty}^{+\infty} S_{p p}(a, t) d t \\
& =\int_{-\infty}^{+\infty}\left|\frac{1}{\sqrt{a}} \psi\left(\frac{b_{k}-t}{a}\right)\right|^{2} S_{f f}(a) d t .
\end{aligned}
$$

Considering several samples of the process $f(t)$, the EPSD of different samples may be different. The mathematical expectation, which can roughly represent the average value of a large number of samples with the same probability distribution, is employed to describe different samples belonging to

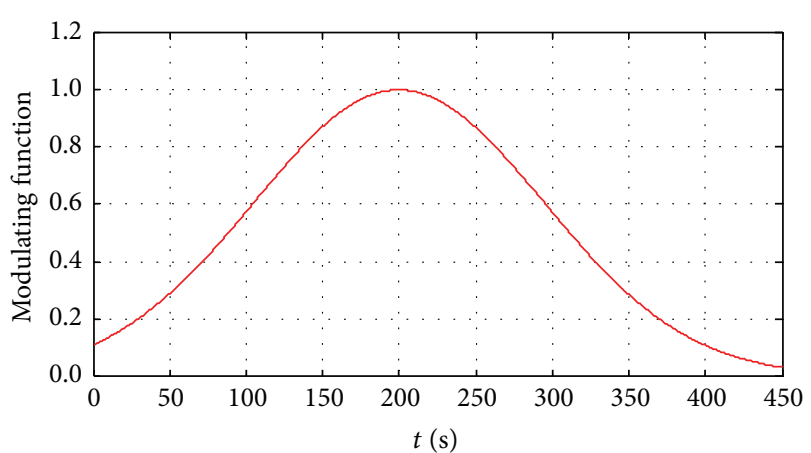

FIGURE 1: Uniformly modulating function $h(t)$.

a typhoon process. Setting mathematical expectation in the left-hand side of (20) derives

$$
E\left[\left|\operatorname{WT}_{f}\left(a, b_{k}\right)\right|^{2}\right]=\int_{-\infty}^{+\infty}\left|\frac{1}{\sqrt{a}} \psi\left(\frac{b_{k}-t}{a}\right)\right|^{2} S_{f f}(a, t) d t .
$$

The general solution to (21) takes the following form:

$$
S_{f f}(a, t)=\sum_{k=1}^{n} \gamma_{k}(a)\left|\frac{1}{\sqrt{a}} \psi\left(\frac{b_{j}-t}{a}\right)\right|^{2} \quad j=1,2, \ldots n,
$$

where $\gamma_{k}(a)$ is the frequency-dependent coefficient. Substituting (22) into (21), the following equation can be made:

$$
\begin{aligned}
& E\left[\left|\mathrm{WT}_{f}\left(a, b_{k}\right)\right|^{2}\right] \\
& =\sum_{k=1}^{n} \gamma_{k}(a) \int_{-\infty}^{+\infty}\left|\frac{1}{\sqrt{a}} \psi\left(\frac{b_{k}-t}{a}\right)\right|^{2}\left|\frac{1}{\sqrt{a}} \psi\left(\frac{b_{j}-t}{a}\right)\right|^{2} d t \\
& =\sum_{k=1}^{n} \gamma_{k}(a) \delta_{k, j} .
\end{aligned}
$$

To sum up, the EPSD of the process $f(t)$ can be calculated by (22). This equation can be explained as that the EPSD is represented as a sum of time-dependent shape functions modulated by frequency-dependent coefficients. The shape functions are the squared moduli of wavelet functions in time domain during the whole duration, and the coefficients relate to the squared values of wavelet transform results and two wavelet functions with different time shifts. Comparing (22) with (24) in [24], the difference is obvious although the format of the two expressions is similar. In this paper, the frequency-dependent coefficients are determined at each frequency point and the EPSD is constructed by wavelet functions in time domain. But, in [24], the time-dependent coefficients are determined at each time instant and the EPSD is constructed by wavelet functions in frequency domain. Therefore, combining the method proposed in this paper with the equation in [24], the EPSD can be estimated by 


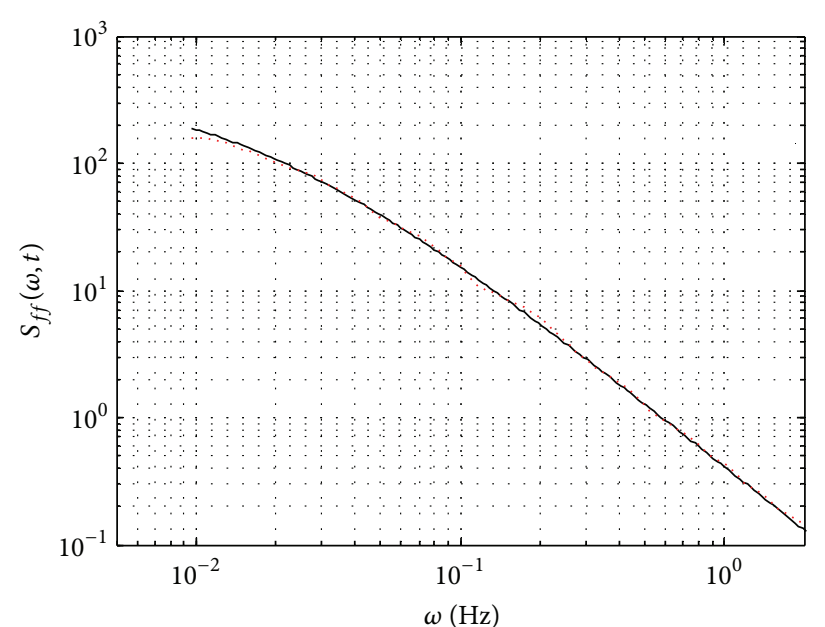

- Theoretical solution Estimated result

(a) $t=125 \mathrm{~s}$



(c) $\omega=0.05 \mathrm{~Hz}$

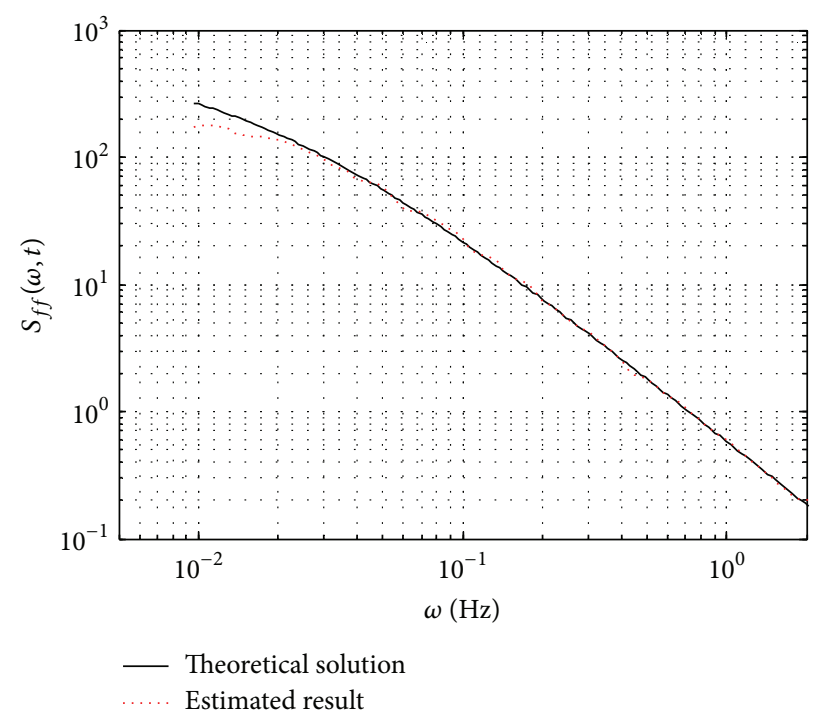

(b) $t=250 \mathrm{~s}$

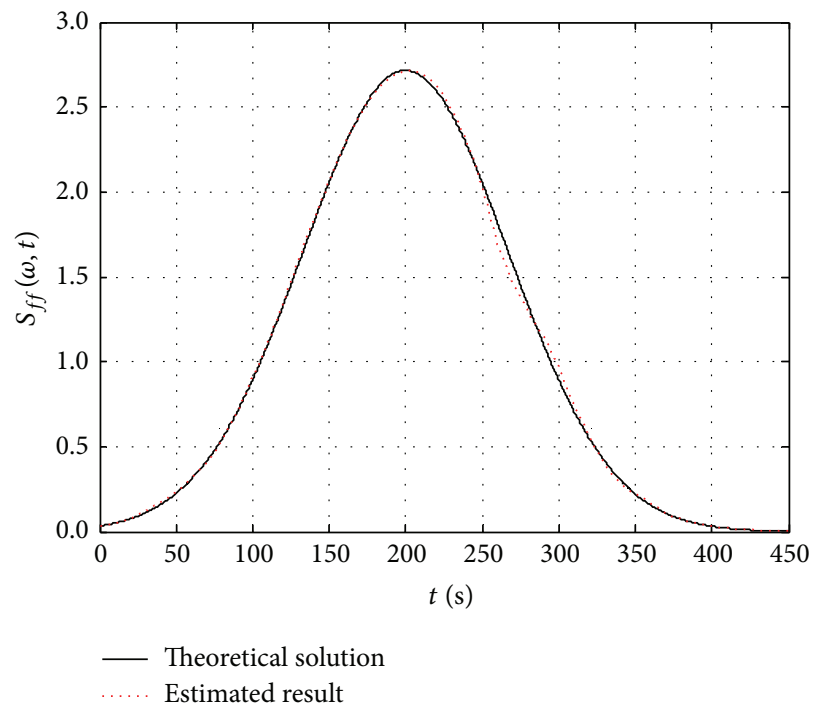

(d) $\omega=0.46 \mathrm{~Hz}$

FIGURE 2: Comparison of estimated EPSD of uniformly modulated typhoon process with theoretical solution.

various wavelets whether their analytical expressions exist in time or frequency domain. The procedure of the EPSD estimation is as follows: (1) implementing wavelet transform to obtain wavelet coefficients with selected scales and different time instants; (2) establishing the frequency-dependent coefficients by solving (23) for all scales; (3) assembling the frequency-dependent coefficients and time-dependent wavelet functions to calculate the EPSD of the process $f(t)$. It should be noted that all theoretical formulations are originated from the definition of the EPSD, which is introduced to describe a class of nonstationary oscillatory processes. Therefore, the results in this paper are only applicable in oscillatory processes. When employing the wavelet transform, only the definition of the wavelet transform is used and the function of wavelet is not specified. So, in theory, the method proposed in this paper is applicable to any wavelet whose function in time domain exists.

\section{Numerical Example}

3.1. Wavelet Function. From (22) and (23), it can be concluded that the accuracy of the wavelet-based EPSD relies heavily on the wavelet function. So, an appropriate wavelet function should be selected before carrying out EPSD estimation. Since large-scale structures are susceptible to the energy distribution of excitation in both time and frequency domains, the high time resolution and frequency resolution of wavelet functions are required. However, according to the Heisenberg uncertainty principle, the time resolution and the frequency resolution are inconsistent. It is impossible 




(a) Marginal feature in time domain

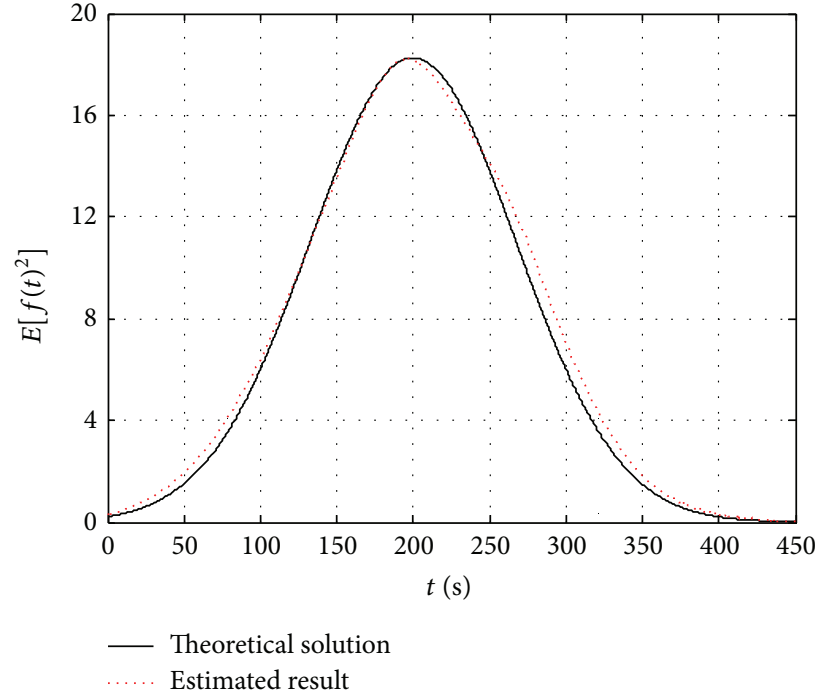

(b) Marginal feature in frequency domain

FIGURE 3: Comparison of marginal features of estimated EPSD with theoretical solution.

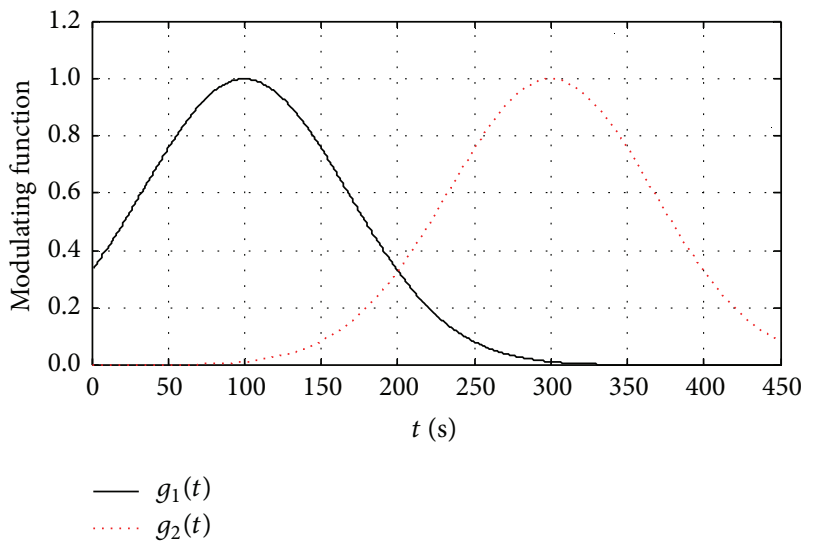

FIGURE 4: Uniformly modulating functions $h_{1}(t)$ and $h_{2}(t)$.

that a wavelet function has highest resolution in both time and frequency domains simultaneously, so only the compromise solution is available. In this paper, the Morlet wavelet, which has acceptable resolution in both time and frequency domains, is adopted to estimate the EPSD of the typhoon process. Its expressions in time domain and frequency domain are

$$
\begin{gathered}
\psi(t)=e^{-t^{2} / 2} e^{i \omega t}, \\
\Psi(\omega)=\sqrt{2 \pi} e^{-\left(\omega-\omega_{0}\right)^{2} / 2},
\end{gathered}
$$

where $\omega_{0}$ represents a parameter that involves the center frequency of the time-frequency window.
3.2. Uniformly Modulated Nonstationary Typhoon Process. Early in 1982, Lin and Yang have suggested that the nonstationary turbulence wind can be described by a stationary stochastic process multiplied with a determined modulating function [28]. In this paper, the concept is employed to simulate the nonstationary wind. The Simiu spectra are introduced as the target power spectra of the stationary stochastic wind $g(t)$. The process $g(t)$ can be synthesized by the classical spectral representation method $[29,30]$. Since there is no widely accepted uniformly modulating function describing the typhoon process, at present, the modulating function in earthquake engineering is referred to and the modified formula is supposed to be as follows:

$$
h(t)=\exp \left(\frac{-(t-\alpha)^{2}}{\beta}\right)
$$

where $\alpha$ and $\beta$ are problem specific parameters. In this example, the selected $\alpha$ and $\beta$ are 200 and 18000, respectively; then the sketch of $h(t)$ is shown in Figure 1.

The simulated wind duration and the sampling frequency are set as $450 \mathrm{~s}$ and $4 \mathrm{~Hz}$, respectively. 500 samples of the process $f(t)$ are synthesized and the average values of wavelet coefficients are calculated. Then, the EPSD of simulated typhoon processes is estimated by (22). The estimated EPSD is compared with the theoretical solution to evaluate the reliability of the proposed method. In order to clearly display the results, the three-dimensional EPSD is sliced at different time instants and different frequency points, as shown in Figure 2, which are named as the time slice and the frequency slice in this paper, respectively. In the figure, the unit of frequency is converted to $\mathrm{Hz}$. The comparison between the estimated results and the theoretical solution indicates that 


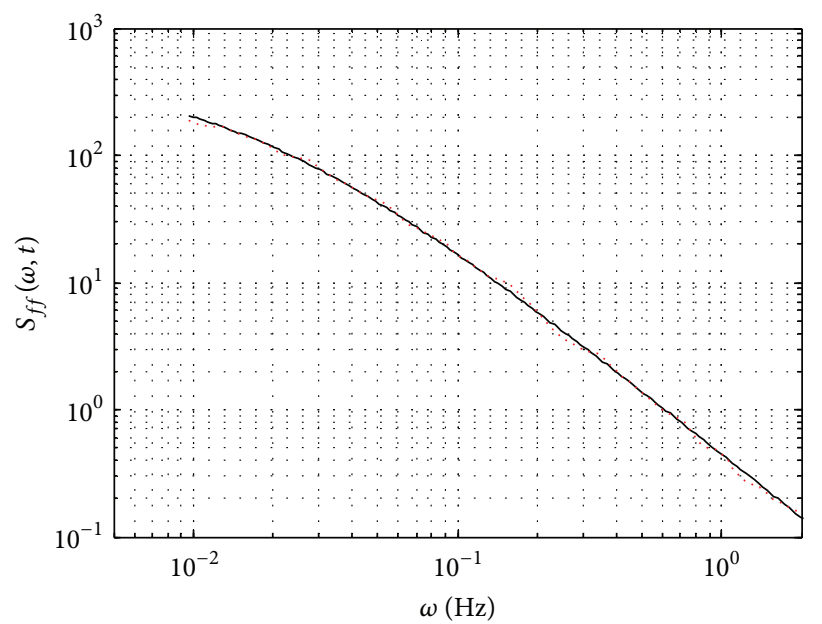

— Theoretical solution Estimated result

(a) $t=10 \mathrm{~s}$

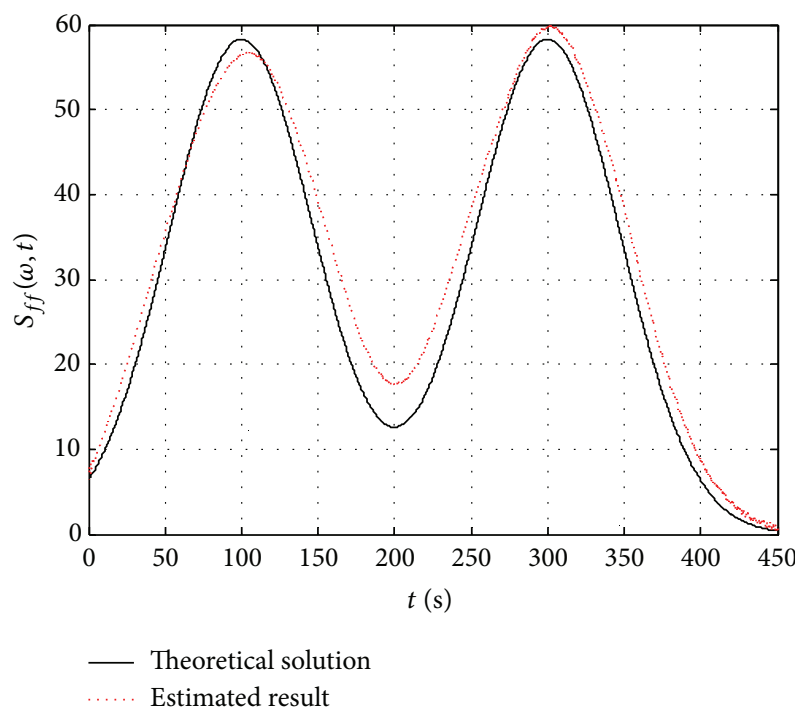

(c) $\omega=0.06 \mathrm{~Hz}$

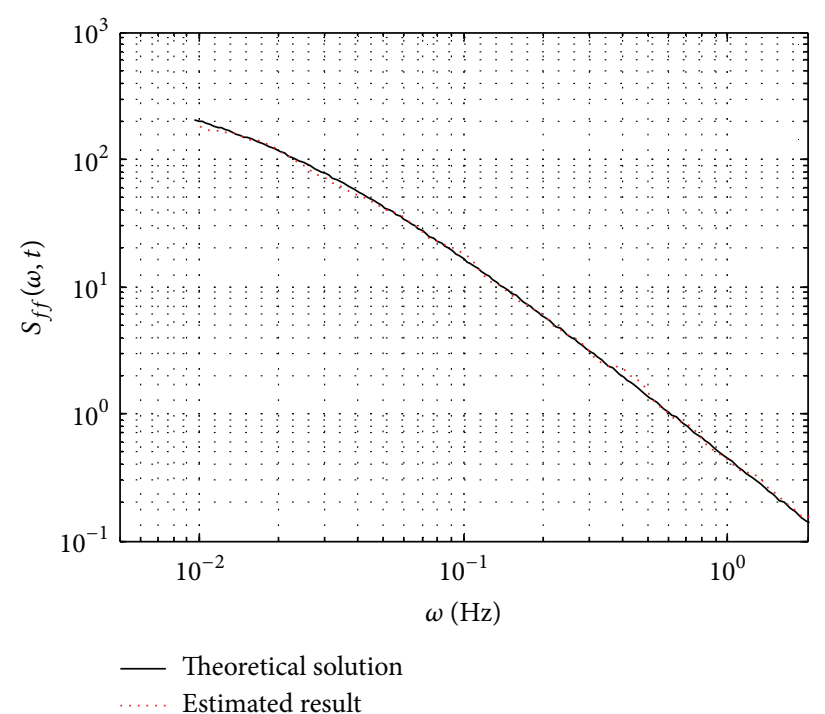

(b) $t=250 \mathrm{~s}$

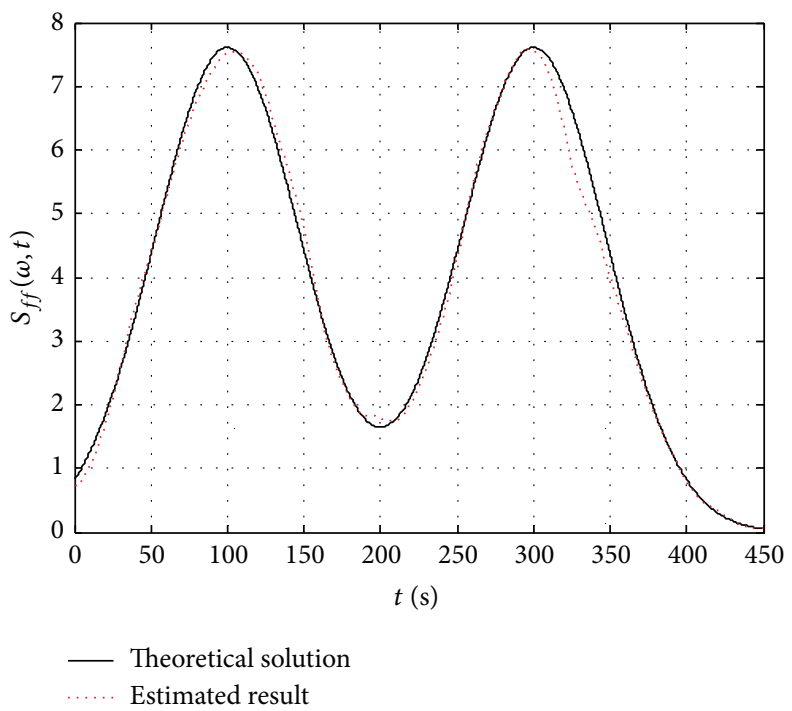

(d) $\omega=0.24 \mathrm{~Hz}$

FIGURE 5: Comparison of estimated EPSD of non-uniformly modulated typhoon process with theoretical solution.

the estimated EPSD has a satisfactory agreement with the theoretical solution in both time and frequency domains although the estimated EPSD may deviate slightly from the theoretical solution at some time instants and frequency points. The effectiveness of the proposed wavelet-based EPSD estimation method is validated. The EPSD extends the two-dimensional power spectral density with energy and frequency to three-dimensional evolutionary power spectral density with energy, frequency, and time. One can investigate the energy distribution of a signal at any time instant and frequency point, which is significant for accurately predicting structural dynamic responses under typhoon processes.

Using the marginal features, the mean-square values of time-independent power spectral density of the nonstationary typhoon process $f(t)$ and the instantaneous meansquare values of the nonstationary typhoon process $f(t)$ are obtained. Figure 3 displays the comparison between the estimated marginal features and the exact solution. An excellent agreement can be found between the two results in the figure, which again demonstrates that the proposed method is applicable in estimating the EPSD of nonstationary typhoon processes modulated by the uniformly modulating function.

3.3. Non-Uniformly Modulated Nonstationary Typhoon Process. Suppose the non-uniformly modulated nonstationary typhoon process $f(t)$ is formulated as

$$
f(t)=h_{1}(t) g_{1}(t)+h_{2}(t) g_{2}(t),
$$

where $h_{1}(t)$ and $h_{2}(t)$ are two uniformly modulating functions and $g_{1}(t)$ and $g_{2}(t)$ are two stationary typhoon 


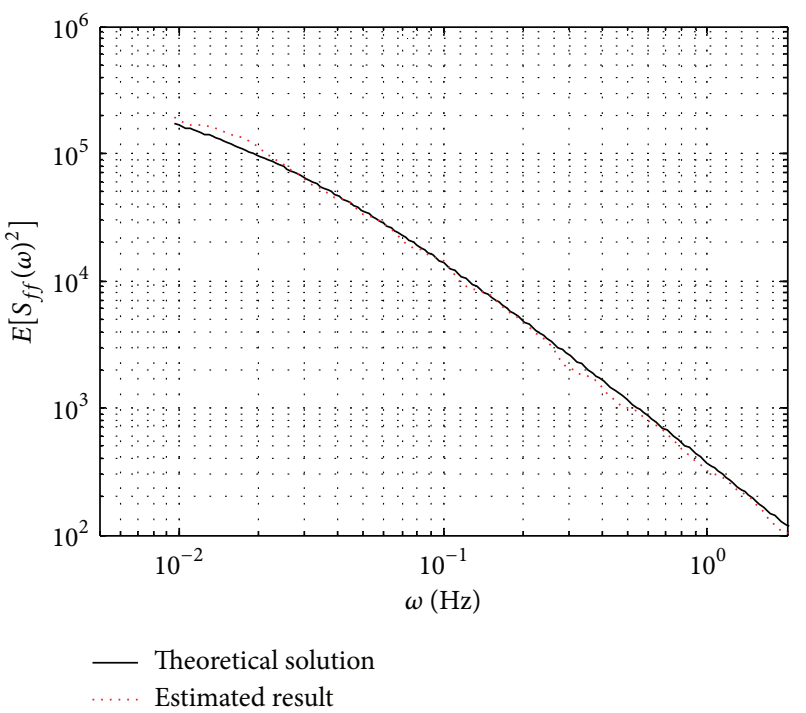

(a) Marginal feature in time domain

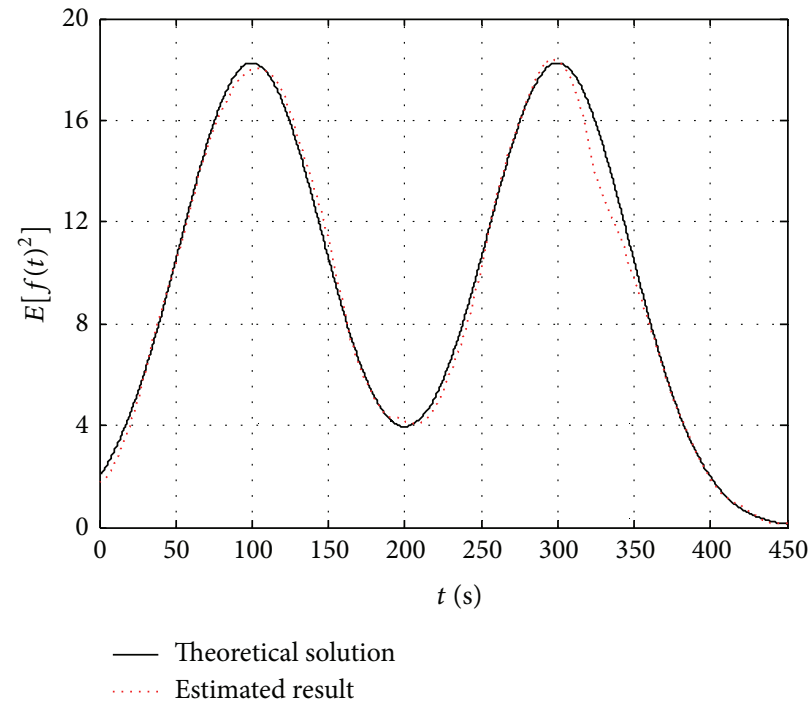

(b) Marginal feature in frequency domain

FIGURE 6: Comparison of marginal features of estimated EPSD with theoretical solution.

processes described by the Simiu spectra. $h_{1}(t)$ and $h_{2}(t)$ are formulated by

$$
\begin{aligned}
& h_{1}(t)=\exp \left(\frac{-\left(t-\alpha_{1}\right)^{2}}{\beta_{1}}\right), \\
& h_{2}(t)=\exp \left(\frac{-\left(t-\alpha_{2}\right)^{2}}{\beta_{2}}\right),
\end{aligned}
$$

where $\alpha_{1}, \alpha_{2}, \beta_{1}$, and $\beta_{2}$ are problem specific parameters. Here, $\alpha_{1}, \alpha_{2}, \beta_{1}$, and $\beta_{2}$ are set as 100, 300, 9000, and 9000, respectively. Figure 4 displays the values of $h_{1}(t)$ and $h_{2}(t)$ changing with time. Then, the EPSD of the non-uniformly modulated nonstationary wind typhoon process $f(t)$ is

$$
S_{f f}(\theta, t)=\left|h_{1}(t)\right|^{2} S_{g_{1} g_{1}}(\theta)+\left|h_{2}(t)\right|^{2} S_{g_{2} g_{2}}(\theta) .
$$

Using the same approach in Section 3.2, the nonuniformly modulated nonstationary typhoon process $f(t)$ is synthesized and the EPSD of $f(t)$ is estimated. The comparison of time slices and frequency slices between the estimated results and the exact solutions is illustrated in Figure 5. In most cases, as displayed in Figures 5(a), 5(b), and 5(d), the estimated results agree well with the exact solutions. Since it is impossible to narrow the window of Morlet function to a frequency point, there are some derivations in the time slices, as shown in Figure 5(c). But the proposed method is successful to capture the peak values of the EPSD, which may induce intensive structural responses; therefore, the estimated EPSD can be used in practical application and the big errors in the valley are acceptable in structural dynamic analysis. The proposed wavelet-based method is effective to estimate nonstationary typhoon processes modulated by the non-uniformly modulating function.

The marginal features of the EPSD of non-uniformly modulated nonstationary typhoon processes are also calculated. The comparison results between the estimated values and the theoretical solutions are shown in Figure 6. The reasonable agreement can also be found, which validates the capability of the proposed method in capturing the timefrequency properties of nonstationary typhoon processes.

\section{Conclusions}

Comprehensively understanding the energy distribution of typhoon processes in both time and frequency domains is significant for reasonably predicting typhoon-induced structural responses. The nonstationary behaviors are found in a large number of field measured typhoon processes, which is different from conventional perspective. The EPSD is a powerful index to describe the time-frequency properties of nonstationary typhoon processes, which belong to the class of nonstationary oscillatory processes. A wavelet-based EPSD estimation method is proposed in this paper. Some conclusions are drawn as follows.

(1) Using the definitions of the continuous wavelet transform and the EPSD, the closed-form expressions used to compute the EPSD are rigorously deduced. The EPSD of nonstationary oscillatory processes can be represented as the weighted sum of the square moduli of wavelet functions in time domain. The weighted coefficients are frequency-dependent and determined by wavelet coefficients and the overlapping area of two wavelets at different time instants. So, the EPSD can be estimated only by wavelet functions in time domain and wavelet coefficients.

(2) The proposed wavelet-based EPSD estimation method is verified by two numerical examples, which employ the stationary typhoon processes modulated by the uniformly modulating function and the non-uniformly modulating function. The results 
indicate that the accuracy of the EPSD estimated by the proposed wavelet-based method is adequate for predicting structural typhoon-induced responses. The proposed wavelet-based method is applicable in the EPSD estimation of nonstationary typhoon processes and can be extended to other nonstationary oscillatory processes.

The nonstationary features of signals are important but rather complex. This paper is only a preliminary research for nonstationary typhoon processes. The investigation of nonstationary typhoon processes and nonstationary windinduced vibrations may be a promising topic in future works.

\section{Conflict of Interests}

The authors declare that there is no conflict of interests regarding the publication of this paper.

\section{Acknowledgments}

The authors wish to gratefully acknowledge Professor T. H. Yi for the valuable suggestions and kindly sharing part codes of wavelet transform. This research work was jointly supported by the 973 Program (Grant no. 2015CB060000), the National Natural Science Foundation of China (Grant nos. 51278104 and 51178100), and the Traffic Science Foundation of Jiangsu Province of China (Grant no. 2011Y03).

\section{References}

[1] L. Hu, Y.-L. Xu, and W.-F. Huang, "Typhoon-induced nonstationary buffeting response of long-span bridges in complex terrain," Engineering Structures, vol. 57, pp. 406-415, 2013.

[2] K. Gurley and A. Kareem, "Applications of wavelet transforms in earthquake, wind and ocean engineering," Engineering Structures, vol. 21, no. 2, pp. 149-167, 1999.

[3] E. Wigner, "On the quantum correction for thermodynamic equilibrium," Physical Review, vol. 40, no. 5, pp. 749-759, 1932.

[4] J. Ville, "Theorie et applications de la notion de signal analytique," Cables and Transmission, vol. 2, pp. 61-74, 1948.

[5] G. Failla, M. Pappatico, and G. A. Cundari, "A wavelet-based spectrum for non-stationary processes," Mechanics Research Communications, vol. 38, no. 5, pp. 361-367, 2011.

[6] R. G. Stockwell, L. Mansinha, and R. P. Lowe, "Localization of the complex spectrum: the S transform," IEEE Transactions on Signal Processing, vol. 44, no. 4, pp. 998-1001, 1996.

[7] R. G. Stockwell, S-transform analysis of gravity wave activity from a small scale network of airglow imagers [Ph.D. thesis], University of Western Ontario, London, Canada, 1999.

[8] T.-H. Yi, H.-N. Li, and M. Gu, "Wavelet based multi-step filtering method for bridge health monitoring using GPS and accelerometer," Smart Structures and Systems, vol. 11, no. 4, pp. 331-348, 2013.

[9] S. G. Mallat, "A theory for multiresolution signal decomposition: the wavelet representation," IEEE Transactions on Pattern Analysis and Machine Intelligence, vol. 11, no. 7, pp. 674-693, 1989.

[10] T.-H. Yi, H.-N. Li, and X.-Y. Zhao, "Noise smoothing for structural vibration test signals using an improved wavelet thresholding technique," Sensors, vol. 12, no. 8, pp. 11205-11220, 2012.

[11] A. Olivero, B. Torresani, and R. Kronland-Martinet, "A class of algorithms for time-frequency multiplier estimation," IEEE Transactions on Audio, Speech and Language Processing, vol. 21, no. 8, pp. 1550-1559, 2013.

[12] T.-H. Yi, H.-N. Li, and M. Gu, "Recent research and applications of GPS-based monitoring technology for high-rise structures," Structural Control and Health Monitoring, vol. 20, no. 5, pp. 649670, 2013.

[13] S. Marchesiello, S. Bedaoui, L. Garibaldi, and P. Argoul, “Timedependent identification of a bridge-like structure with crossing loads," Mechanical Systems and Signal Processing, vol. 23, no. 6, pp. 2019-2028, 2009.

[14] M. Ülker-Kaustell and R. Karoumi, "Application of the continuous wavelet transform on the free vibrations of a steel-concrete composite railway bridge," Engineering Structures, vol. 33, no. 3, pp. 911-919, 2011.

[15] Q. Fei and X. Han, "Identification of modal parameters from structural ambient responses using wavelet analysis," Journal of Vibroengineering, vol. 14, no. 3, pp. 1176-1186, 2012.

[16] W.-X. Ren, X.-X. Xu, and W.-J. Yan, "Operational modal parameter identification based on covariance-driven continuous wavelet transform and singular value decomposition," Advances in Structural Engineering, vol. 16, no. 3, pp. 579-592, 2013.

[17] Z. H. Min and L. M. Sun, "Wavelet-based structural modal parameter identification," Structural Control and Health Monitoring, vol. 20, no. 2, pp. 1-18, 2013.

[18] D. S. Li, Q. Hu, J. P. Ou, and H. Li, "Fatigue damage characterization of carbon fiber reinforced polymer bridge cables: wavelet transform analysis for clustering acoustic emission data," Science China Technological Sciences, vol. 54, no. 2, pp. 379-387, 2011.

[19] D. Hester and A. González, "A wavelet-based damage detection algorithm based on bridge acceleration response to a vehicle," Mechanical Systems and Signal Processing, vol. 28, pp. 145-166, 2012.

[20] K. V. Nguyen, "Comparison studies of open and breathing crack detections of a beam-like bridge subjected to a moving vehicle," Engineering Structures, vol. 51, pp. 306-314, 2013.

[21] B. Basu and V. K. Gupta, "Non-stationary seismic response of MDOF systems by wavelet transform," Earthquake Engineering \& Structural Dynamics, vol. 26, no. 12, pp. 1243-1258, 1997.

[22] B. Basu and V. K. Gupta, "Seismic response of SDOF systems by wavelet modeling of nonstationary processes," Journal of Engineering Mechanics, vol. 124, no. 10, pp. 1142-1150, 1998.

[23] J. Iyama and H. Kuwamura, "Application of wavelets to analysis and simulation of earthquake motions," Earthquake Engineering \& Structural Dynamics, vol. 28, no. 2, pp. 255-272, 1999.

[24] P. D. Spanos and G. Failla, "Evolutionary spectra estimation using wavelets," Journal of Engineering Mechanics, vol. 130, no. 8, pp. 952-960, 2004.

[25] T. Kijewski and A. Kareem, "Wavelet transforms for system identification in civil engineering," Computer-Aided Civil and Infrastructure Engineering, vol. 18, no. 5, pp. 339-355, 2003.

[26] M. B. Priestley, "Power spectral analysis of non-stationary random processes," Journal of Sound and Vibration, vol. 6, no. 1, pp. 86-97, 1967.

[27] A. Moghtaderi, G. Takahara, and D. J. Thomson, "Evolutionary spectrum estimation for uniformly modulated processes with 
improved boundary performance," in Proceedings of the IEEE International Conference on Acoustics, Speech, and Signal Processing (ICASSP '09), pp. 2993-2996, Taipei, Taiwan, April 2009.

[28] Y. K. Lin and J. N. Yang, "Multimode bridge response to wind excitations," Journal of Engineering Mechanics, vol. 109, no. 2, pp. 586-603, 1983.

[29] M. Shinozuka and C.-M. Jan, "Digital simulation of random processes and its applications," Journal of Sound and Vibration, vol. 25, no. 1, pp. 111-128, 1972.

[30] M. Shinozuka, C.-B. Yun, and H. Seya, "Stochastic methods in wind engineering," Journal of Wind Engineering \& Industrial Aerodynamics, vol. 36, no. 1-3, pp. 829-843, 1990. 


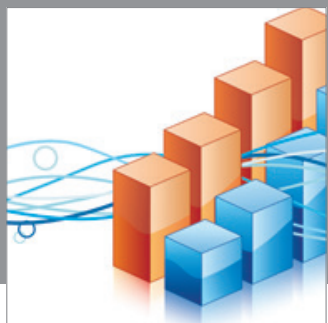

Advances in

Operations Research

mansans

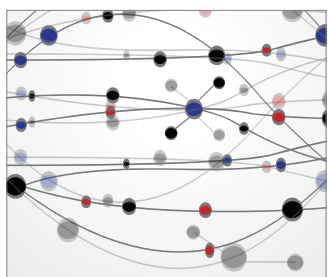

The Scientific World Journal
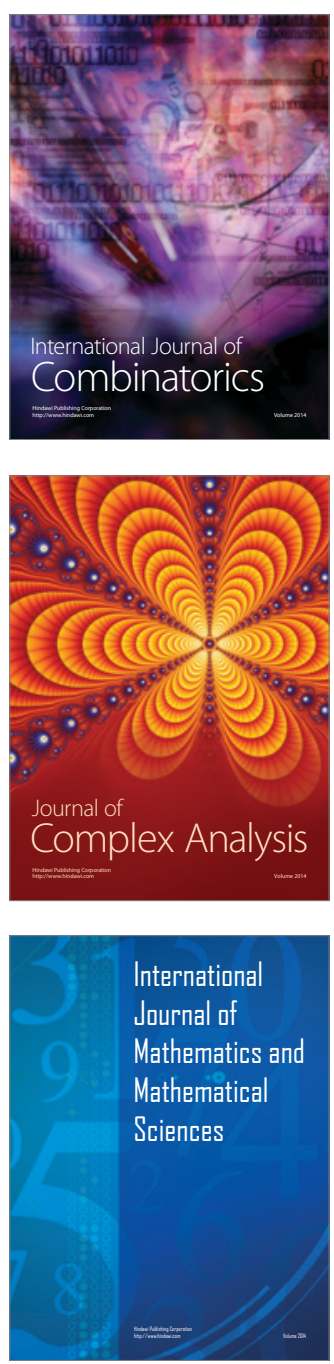
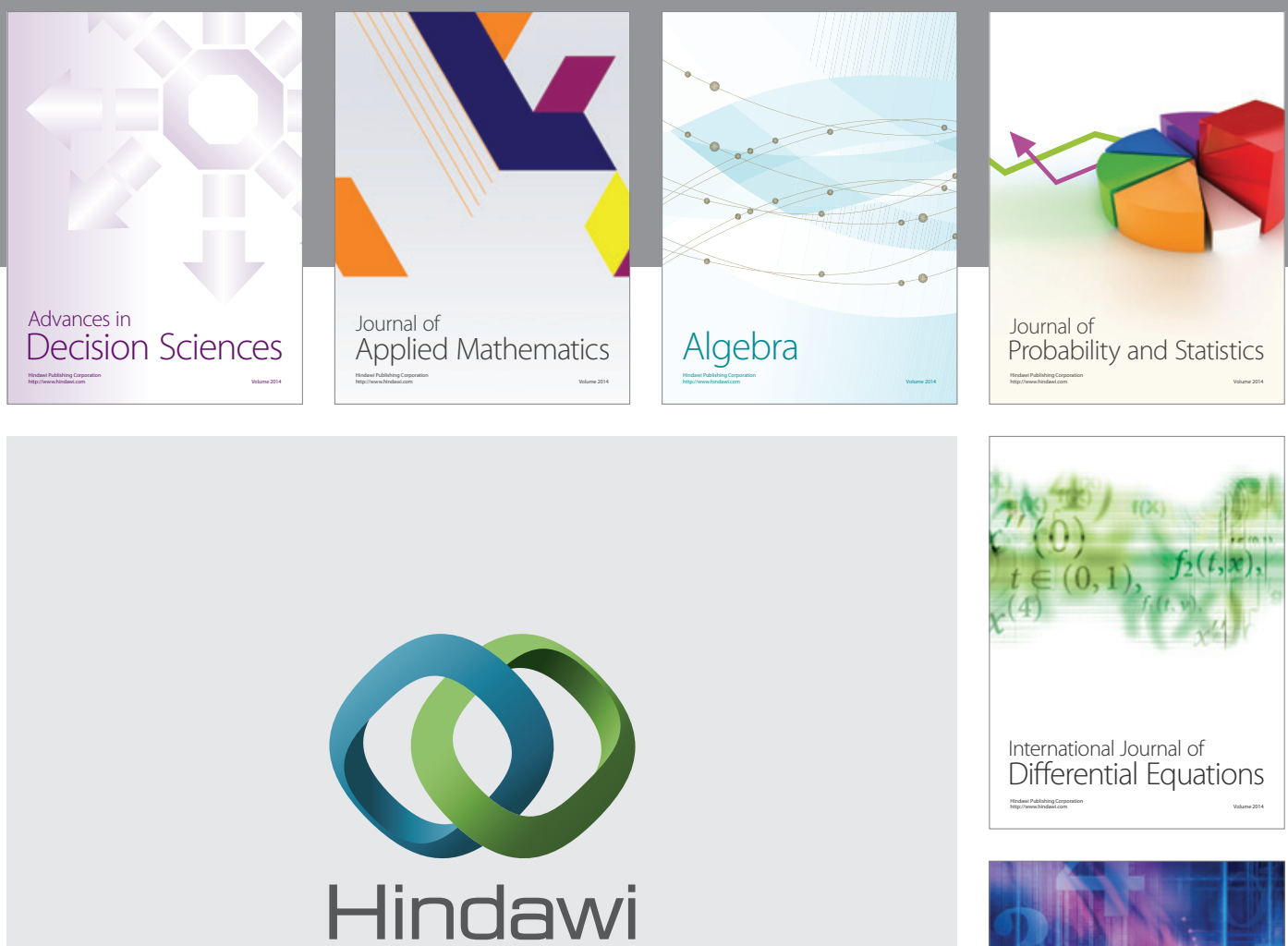

Submit your manuscripts at http://www.hindawi.com
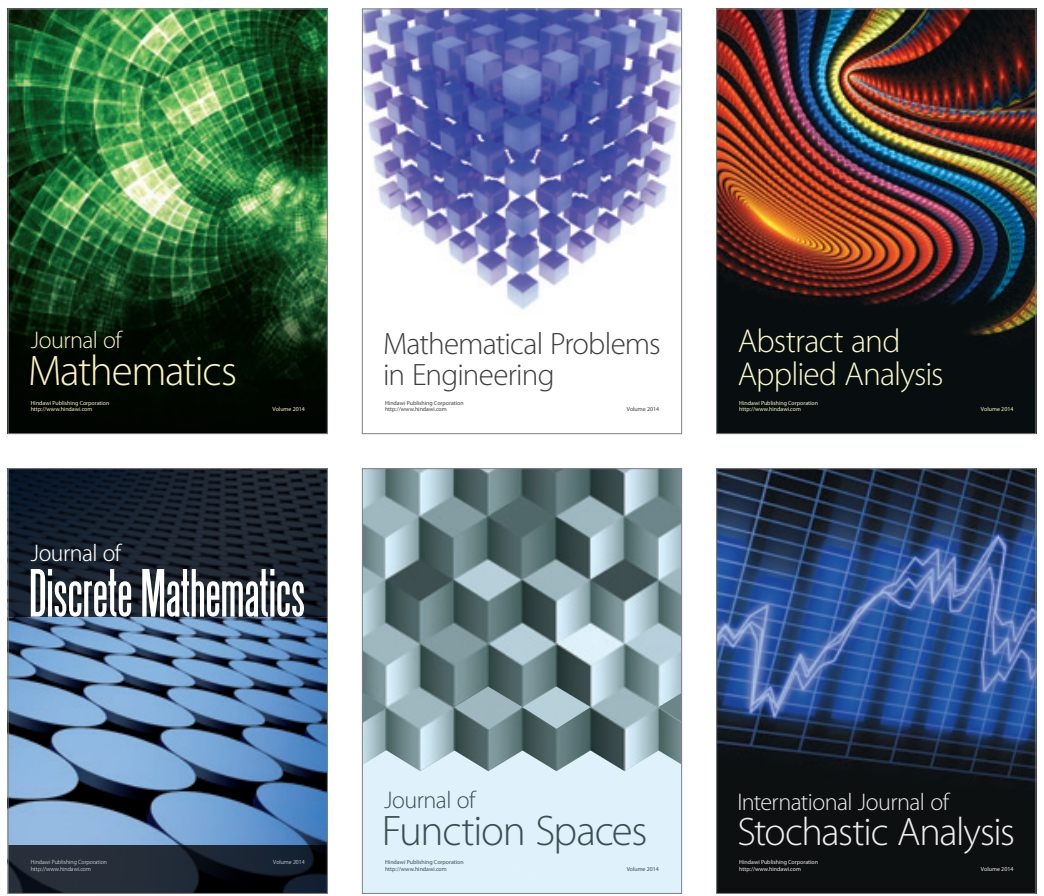

Journal of

Function Spaces

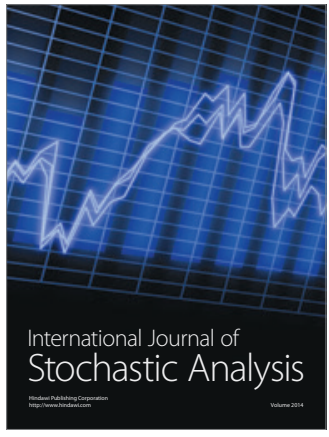

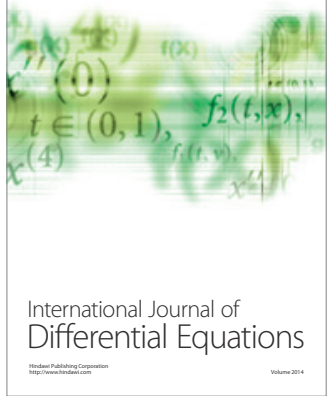
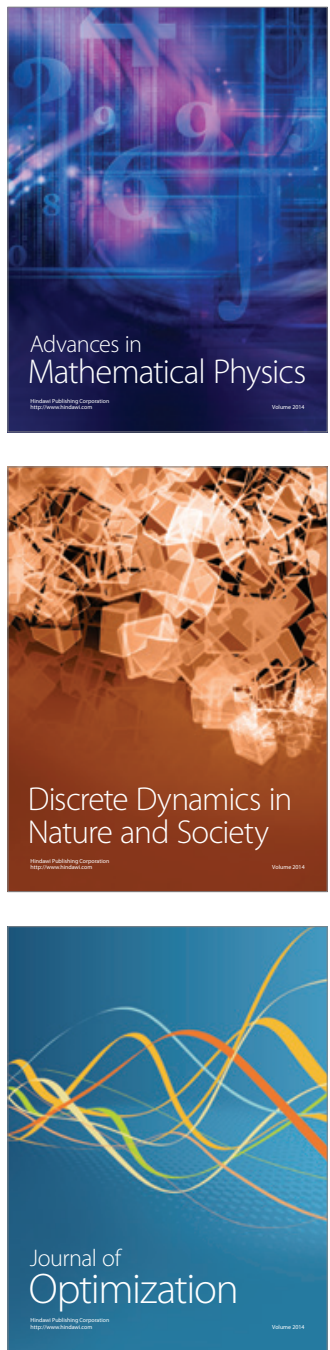